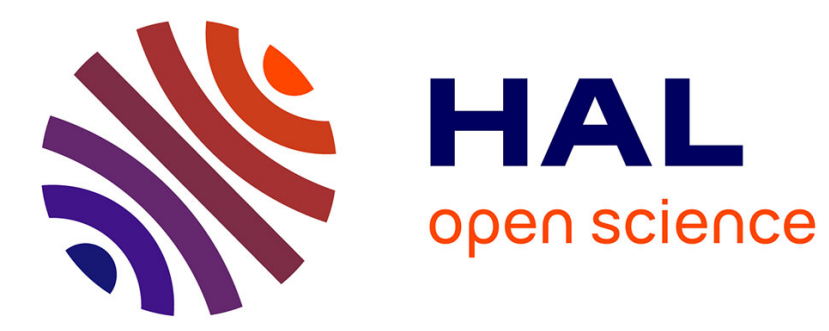

\title{
La figure de l'enfant mort entre fantôme et fantasme Mi-Kyung Yi
}

\section{To cite this version:}

Mi-Kyung Yi. La figure de l'enfant mort entre fantôme et fantasme. Cliniques méditerranéennes, 2012, L'enfant et ses fantômes, 2 (86), pp.9 - 20. 10.3917/cm.086.0009 . hal-01524830

\section{HAL Id: hal-01524830 \\ https://hal.science/hal-01524830}

Submitted on 2 Jun 2017

HAL is a multi-disciplinary open access archive for the deposit and dissemination of scientific research documents, whether they are published or not. The documents may come from teaching and research institutions in France or abroad, or from public or private research centers.
L'archive ouverte pluridisciplinaire HAL, est destinée au dépôt et à la diffusion de documents scientifiques de niveau recherche, publiés ou non, émanant des établissements d'enseignement et de recherche français ou étrangers, des laboratoires publics ou privés. 


\title{
LA FIGURE DE L'ENFANT MORT ENTRE FANTÔME ET FANTASME Mi-Kyung Yi
}

\author{
ERES | « Cliniques méditerranéennes »
}

$2012 / 2 n^{\circ} 86 \mid$ pages 9 à 20

ISSN 0762-7491

ISBN 9782749234359

Article disponible en ligne à l'adresse :

http://www.cairn.info/revue-cliniques-mediterraneennes-2012-2-page-9.htm

\section{!Pour citer cet article :}

Mi-Kyung Yi, «La figure de l'enfant mort entre fantôme et fantasme », Cliniques méditerranéennes 2012/2 ( $\left.{ }^{\circ} 86\right)$, p. 9-20.

DOI $10.3917 / \mathrm{cm} .086 .0009$

Distribution électronique Cairn.info pour ERES.

(C) ERES. Tous droits réservés pour tous pays.

La reproduction ou représentation de cet article, notamment par photocopie, n'est autorisée que dans les limites des conditions générales d'utilisation du site ou, le cas échéant, des conditions générales de la licence souscrite par votre établissement. Toute autre reproduction ou représentation, en tout ou partie, sous quelque forme et de quelque manière que ce soit, est interdite sauf accord préalable et écrit de l'éditeur, en dehors des cas prévus par la législation en vigueur en France. Il est précisé que son stockage dans une base de données est également interdit. 


\section{Mi-Kyung Yi}

\section{La figure de l'enfant mort entre fantôme et fantasme}

L'ombre de l'enfant mort plane dans diverses situations cliniques, en particulier dans les problématiques inhérentes aux dépressions et à la psychopathologie transgénérationnelle. Là, l'idée du fantôme semble s'imposer d'elle-même, tant certains paysages psychiques en sont lourdement imprégnés, comme celui de l'enfant dit de remplacement. Or, en psychanalyse, on parle autant sinon davantage de la figure de l'enfant mort. Une façon de marquer l'écart entre le mot et la réalité désignée, de soutenir la multiplicité des représentations associées, lesquelles débordent la réalité événementielle de la perte. Prenons l'exemple de la figure de l'enfant mort, celle qu'on devine à l'œuvre dans des dépressions graves. À vouloir y reconnaître uniquement la manifestation de l'emprise d'un fantôme hors jeu psychique, on manquerait la part de fantasme narcissique, celui qui tend à l'abolition de tout mouvement, de toute limite, à commencer par celle entre vie et mort. L'enfant mort figure le paradoxe d'une psyché résignée à rester encore vivante, le temps de chercher à mourir pour toujours. Parce que essentiellement elle oscille entre fantôme et fantasme, la figure de l'enfant mort offre une voie d'approche de la fonction et du statut de métaphore que la psychanalyse accorde au fantôme.

\section{LE FANTÔME SERAIT-IL DEVENU L'OMBRE DE LUI-MÊME ?}

« L'air est maintenant tout empli d'un tel fantôme ${ }^{1}$. » C'est par ces mots, empruntés au Faust de Goethe, que Freud se plaît à annoncer l'avènement de la psychopathologie de la vie quotidienne. Nous sommes en 1901, soit un an

Mi-Kyung Yi, maître de conférences en psychopathologie, UFR sciences humaines cliniques, université Paris 7, Paris Diderot, psychanalyste ; 34 rue Desnouettes, 75015 Paris-mik.yi@wanadoo.fr

1. Lettre à Fliess n 139 du 14 oct. 1900. 
après la publication de L'interprétation des rêves. La production nocturne vient de conquérir le statut d'activité psychique à part entière. À la lumière de l'expérience analytique, les nuits s'éclairent, tandis que paradoxalement, la vie éveillée révèle sa part d'ombre. La frontière entre le jour et la nuit, comme celle entre l'esprit sain et l'esprit dérangé, s'en trouve irrémédiablement brouillée et continuellement déplaçable. L'inconscient n'est plus l'apanage de la pathologie, ni le privilège de la " psychose onirique », mais devient affaire de la vie ordinaire. Pareil à un arpenteur clandestin et infatigable, l'inconscient traverse et sillonne le territoire de la vie psychique commune en provoquant sur son passage des accidents, un acte manqué ici, un lapsus là, tantôt un sentiment de trouble insaisissable, tantôt une image, une idée intense, sans queue ni tête. N'est-il pas en effet comme un fantôme errant, qui hante la vie de tous les jours ? Un fantôme d'autant plus étrange qu'il surgit inopinément, qu'il semble si actif, si vif. De son apparition aussi inquiétante que méconnaissable, la psyché - si elle est relativement assurée de ses assises - se défend alors sur le mode de : «Le fantôme? Je n'y crois pas mais j'en ai peur ${ }^{2} . .$. »

Croire aux fantômes, au risque même de courir après : telle est aussi une des critiques régulièrement adressées à la psychanalyse, celle d'être passéiste, de revenir constamment vers le passé. On se souvient de Jung qui reprochait à la méthode freudienne de ramener tout au passé infantile, au lieu de s'intéresser au présent et de se tourner vers le futur. Le reproche peut aussi surgir à l'intérieur d'un traitement analytique, sur le mode d'une plainte ou d'une crainte. Une patiente se demande si elle n'est pas en train de "s'enterrer dans son passé ». Idée angoissante jusqu'à la suffocation : elle voulait faire une analyse, non pas à la recherche du temps perdu mais plutôt pour se défaire du passé qui ne la lâche pas. Or, l'enseignement principal de l'expérience analytique, immanquablement renouvelé et actualisé dans chaque cure, c'est que le temps ne passe pas, que le passé est présent, selon l'expression de Pontalis ${ }^{3}$. Il s'agit même de la découverte fondatrice de l'invention freudienne, méconnue, voire refoulée précisément par tous ceux tentés de réduire l'expérience analytique à une nostalgie anachronique pour le passé décrété révolu.

Retour donc au temps de la psychanalyse naissante, à l'heure des Études sur l'hystérie. C'est de réminiscences que souffre l'hystérique, disait Freud pour souligner la singularité inouïe du souvenir prétendument oublié. Exclu de la vie psychique consciente, coupé de ses origines, rendu erratique, ce fragment de mémoire s'avère étonnamment conservé dans toute sa fraîcheur et toute son efficience : aussi vivace et chargé d'affects et aussi agissant qu'au moment de son événement, comme s'il était soustrait à l'usure du temps ;

2. Selon le bon mot de la marquise du Deffand.

3. J.-B. Pontalis, Ce temps qui ne passe pas, Paris, Gallimard, 1997. 
ainsi son surgissement à la conscience peut-il revêtir une réalité quasi hallucinatoire. À la manière d'une apparition, pourrait-on dire. Mais ce n'est pas au titre d'un passé maintenu et retrouvé intact par-delà des ans écoulés que les « réminiscences » sollicitent, chez Freud, la métaphore du fantôme, mais davantage comme une force vive et active dans le temps présent. Ce qui est refoulé ne reste pas simplement enfoui dans les profondeurs psychiques, en attente d'être exhumé, mais fait retour, comme un fantôme qui cherche à reprendre vie. Comment ne pas songer ici à la Gradiva redidiva, dans laquelle se trouve incarné l'objet du désir sexuel infantile refoulé du jeune archéo$\operatorname{logue}^{4}$. Or, l'attrait de la jeune fille antique sortie de terre et revenue à la vie, on se souvient, ne réside pas seulement dans sa résurgence hallucinatoire, mais dans sa démarche qui entraîne, sur la trace de ses pas, la vie jusque-là figée, inanimée comme les pierres. Gradiva, celle qui marche victorieusement vers le combat sous le signe du Mars Gravidus, dé-loge et dé-place! Plus que comme une pâle apparition, l'inconscient refoulé agit ainsi comme un « revenant » qui exige son dû : ça revient on ne sait d'où, ça veut on ne sait quoi, ça pousse vers on ne sait quelle destination.

Une exigence in-quiétante - on n'est jamais à l'abri de sa force dé-liante, dé-chaînante - mais aussi excitante et mobilisatrice de l'activité psychique. Le petit Hans raconte au père, dans la suite de son fantasme des deux girafes : "J'ai été avec toi à Schönbrunn voir les moutons, alors nous nous sommes glissés sous les cordes et alors nous l'avons dit à l'agent à l'entrée du jardin et il nous a empoignés. » Le père y entend simplement le désir de faire quelque chose d'interdit et reste sourd à un autre désir plus obscur, celui de faire quelque chose d'interdit avec maman, comme le fait le père lui-même. L'enfant invente alors une autre histoire qui en quelque sorte aggrave leur cas et l'excitation monte d'un cran : " Je suis allé en chemin de fer avec toi et nous avons brisé une fenêtre et l'agent nous a emmenés. » Commentaire de Freud : «Ce qui est ainsi incompris revient. Cela n'a pas de repos, tel un esprit non absous, jusqu'à ce que cela ait accédé à la solution (Lösung) et l'absolution (Erlösung ${ }^{5}$ ). » Le désir inconscient travaille la psyché, met en branle l'activité fantasmatique, à la manière d'un fantôme obstiné.

Fantôme et fantasme. Cette belle trouvaille est, au fond, une retrouvaille. La fonction de métaphore que Freud accorde au "fantôme " pour illustrer l'action ou l'actualité du fantasme (Phantasie) inconscient remet en honneur l'identité de leur source étymologique : le phantasma, qui signifie à la fois vision, illusion, apparition, fantôme. Le mot latin lui-même dérive du grec de la même graphie, lequel connote fortement l'extériorité de la vision en ques-

4. S. Freud (1907), Le délire et les rêves dans la Gradiva de W. Jensen, Paris, Gallimard, 1986.

5. S. Freud (1909), "Analyse de la phobie d'un garçon de cinq ans », dans CEuvres complètes, t. IX, Paris, PUF, 1998, p. 108. 
tion : le phantasma grec est une apparition venue du dehors, surgissant dans le monde du rêve et s'envolant au réveil ${ }^{6}$. Il est également intéressant de noter que lors de son passage du grec au latin, le mot gagne une coloration sexuelle inédite : le phanstama dans son acception latine désigne également, selon Pascal Quignard ${ }^{7}$, le fantôme masturbatoire, le double halluciné qui vient garder les hommes à l'heure des joies esseulées... l'ange gardien des heures sexuelles solitaires, en quelque sorte. Le phantasma comme apparition venue d'ailleurs ou fantôme sexuel accompagnateur. De ces significations originelles, l'emploi freudien du fantasme retrouve une résonance tout actuelle.

La figure du fantôme dans la pensée freudienne n'est pas loin de se confondre tout simplement avec la force motrice de l'inconscient sexuel, avec ce que Freud appelle l'inconscient dynamique. C'est un fantôme qui désire. De cette puissance métaphorique du fantôme, que nous reste-t-il ? Tout ou presque. Si ce n'est que le fantôme a changé, pour ainsi dire. Il évoque moins le mouvement que la matière inerte, moins une présence refoulée et agissante qu'un dépôt enkysté et immobilisant, moins un "revenant » qu'un occupant embaumé. Le fantôme ne figure plus le fantasme mais semble bel et bien installé en lieu et place du fantasme. Autrement dit, il ne représente plus l'action du fantasme inconscient, mais au contraire signe son absence ou son empêchement, voire son impossibilité. Qu'on la sollicite à propos d'un secret inavouable, d'un deuil impossible, d'un traumatisme dénié ou insurmonté, ou d'une position identificatoire assignée ou paradoxale, la figure du fantôme souligne cette forme de hantise accaparante, paralysante, rebelle au mouvement, au déplacement. Impossible à introjecter tout autant qu'impossible à expulser, ce fantôme-là est un " bloc de réalité » encrypté dans la psyché. Un occupant souterrain, une «crypte», pour reprendre une des notions maîtresses des travaux de Nicolas Abraham et Maria Torok ${ }^{8}$.

La référence métapsychologique sollicitée change également. Ce n'est plus l'inconscient dynamique, mais le point de vue topique qui prime. Or, qui dit topique dit point de vue du moi. Il est donc davantage question du lieu psychique, accompagné du retour en force du modèle archéologique. À noter également que la métaphore freudienne du fantôme disparaît paradoxalement de la seconde topique, alors que le retour du même - que signifie la compulsion de répétition - paraîtrait la solliciter presque naturellement... Enfin, à travers l'évolution du statut du fantôme dans les références psychanalytiques, on retrouve le débat sur la réalité psychique du fantasme. Retour du refoulé ou retour du fantôme? La conception du fantasme proposée par N. Abraham et M. Torok autour de la figure de la crypte emprunte davan-

6. P. Quignard, La nuit sexuelle, Paris, Flammarion, 2007, p. 86.

7. P. Quignard, Le sexe et l'effroi, Paris, Gallimard, coll. «Folio », 1994, p. 273.

8. N. Abraham et M. Torok (1978), L'écorce et le noyau, Paris, Flammarion, 1987. 
tage au modèle de la mélancolie, voire au modèle de la perversion, comme en témoigne son insistance sur l'immobilisation, la conservation, la paralysie du processus psychique. C'est dire que la question du fantôme, au-delà de la singularité du champ psychopathologique particulièrement concerné, engage un ensemble de problématiques fondamentales à la théorie et à la pratique psychanalytiques.

Le lien avec l'inconscient sexuel distendu ou rompu, le fantôme serait-il devenu l'ombre de lui-même, irrémédiablement ? Ombre enkystée aux accents mélancoliques, spectre errant sous les auspices du destin, telle est la vision du fantôme particulièrement sensible et imposante dans la clinique des dépressions et la psychopathologie transgénérationnelle. Au croisement de ces deux problématiques se trouve régulièrement repérable, comme un point névralgique, une figure du fantôme : l'enfant mort.

\section{L'ENFANT MORT ENTRE FANTÔME ET FANTASME}

La figure de l'enfant mort s'est trouvée, il y a quelques années, au cœur d'une polémique littéraire opposant deux récits de la mort d'un enfant. L'un inscrit un nom - Philippe - et l'autre porte une phrase - Tom est mort; deux histoires racontées l'une comme l'autre par la mater dolorosa, à ceci près que l'une évoque une réalité insensée vécue par l'auteur elle-même, celle de son bébé mort le jour de sa naissance, et l'autre est une fiction, celle d'une mère plongée dans les affres du deuil indicible et interminable de son enfant mort brutalement à 4 ans. La mère et auteur de Philippe accuse l'auteur de Tom est mort de "plagiat psychique », $\mathrm{d}^{\prime}$ " usurpation $\mathrm{d}^{\prime}$ identité $^{9}$ ». Un point mis en relief dans cette polémique pourrait éveiller un intérêt psychanalytique : de la mort d'un enfant, de ces deux mots scandaleusement réunis, on peut difficilement faire une histoire sans provoquer un malaise, sans faire éprouver une impression, troublante, de transgression. Comme si l'intention narrative portait atteinte à ce qu'il y a là d'intouchable, d'intraitable. Tout au plus, on pourrait en témoigner sous la forme d'un récit qui trouve sa légitimité - unique ou ultime - dans la réalité du malheur subi ${ }^{10}$. Même rien qu'à tenter de réfléchir les maux, il arrive que les mots se voient soupçonnés de s'en

9. « J'ai eu le sentiment, écrit Camille Laurence, en le lisant, que Tom est mort avait été écrit dans ma chambre, le cul sur ma chaise ou vautrée dans mon lit de douleur. Marie Darieussecq s'est invitée chez moi, elle squatte ", propos cité dans « Tom est mort », la polémique, signé Patrick Kéchichian, Le Monde du 24 août 2007.

10. «Je suis mise en demeure, répond Marie Darieussecq, de me justifier pour avoir osé parler de la mort des enfants... On n'écrit pas Tom est mort sans raison. Mes parents ont perdu un enfant. Il y a chez eux une forme de silence que je respecte, admire. Je ne suis pas moins légitime comme sœur que comme mère endeuillée. Il y a une universalité de la douleur », propos cité dans Le Monde, ibid. 
écarter sinon de les trahir : peut-on être dans la douleur et en même temps l'exprimer? Le langage ainsi mis à mal, voire condamné dans sa volonté de signifiance, "frappé de nullité », n'en demeure pas moins sollicité, convoqué par la réalité et la souffrance cramponnées au statut d'ineffable. Jusqu'à la hantise! Il s'ensuit que l'enfant mort hante le langage sans l'habiter, comme il occupe la psyché sans en faire partie. Tel un fantôme...

« Dire que si elle n'était pas morte, tu ne serais pas née... » Nina avait 9 ans quand elle a appris, par cette parole de la mère, l'existence d'une sœur morte à l'âge de 2 ans, à peine un an avant sa naissance. Parole d'autant plus énigmatique, obscure qu'elle est comme laissée tombée de la bouche de la mère dans un moment joyeux de tendresse partagé. Sans doute après un tel drame est-il inévitable que le bonheur présent ou possible traîne, comme son ombre inséparable, la douleur de ce qui est perdu. Pensée douloureuse, mais tolérable pour cette jeune femme portée sur la tristesse, irrésistiblement. Nina cultive ce qu'on appelle mélancolie douce, ce «bonheur d'être triste $^{11}$ » : sa tristesse a un goût de retrouvailles, du moins la chaleur d'une présence. Puis, à l'occasion d'une séance déplacée - ou remplacée selon son expression - surgit sans crier gare une idée autrement vertigineuse, aussitôt repoussée : «Si ma sœur n'était pas morte, je ne serais pas là... comme si ma mère avait dû choisir. " La vocation de Nina, son choix à elle, c'est de s'occuper des enfants orphelins. Une mère de remplacement qui ne serait pas là, si n'était pas... Nina est une mère fraternelle.

La figure de l'enfant mort apparaît en psychanalyse comme une figure polysémique, mais le plus communément elle évoque, comme dans le cas de Nina, l'ombre d'un frère ou d'une sœur arraché à la vie, trop tôt. Trop tôt, parce qu'un enfant ne peut pas, ne doit pas mourir ; chose insensée comme l'aube happée par la nuit. Trop tôt aussi pour qu'un autre puisse venir après : enkysté dans la psyché parentale, l'enfant mort est un rival inégalable parce que indélogeable. Un éternel fidèle, irremplaçable : « Lui, il restera toujours avec moi, jamais il ne me quittera », dit une mère endeuillée. Aussi ne meurt pas l'enfant mort, on ne peut même pas le tuer. C'est un éternel enfant. Nina se surprend à appeler « ma petite sœur » celle envers qui elle se sent coupable d'avoir eu un désir de mort... posthume! Déjà morte avant d'être tuée ? Cela ne change rien au sentiment étrange $d^{\prime}$ ' être pour quelque chose » dans cette disparition, car elle lui doit sa naissance. Comment le temps lui-même n'en perdrait-il pas la tête ? " L'ordre des générations, note J. André, n'est plus ce qu'il était, la suite en est interrompue, désorganisée. La flèche du temps a perdu la boussole, l'avenir c'était hier ${ }^{12}$. »

11. Le mot est de Victor Hugo.

12. J. André, « Le temps mort», dans Folies minuscules, Paris, Gallimard, 2008, p. 76. 
Il arrive également que l'ombre de l'enfant mort occupe les replis de la région psychique sans que l'on puisse la rattacher à une perte reconnaissable. Enfant de personne, n'appartenant à personne, mais installé, par-delà des générations, comme le néant aspirant. Sa silhouette obscure se laisse soupçonner, essentiellement, à travers l'héritage mélancolique marquant le processus identificatoire. Un fantôme errant de génération en génération, qui condamne les vivants à n'en être que le porteur figé ou une stèle commémorative. Aussi insaisissable soit-il, il semble forcer une croyance en sa réalité dans certains états dépressifs et mélancoliques, tant ces pathologies imposent l'image d'une psyché mort vivante : inerte, immobile, réduite à ne rien vouloir, à ne rien attendre, jusqu'à ce que même cette attente du rien s'oublie d'elle-même, disparaisse dans la nuit noire. Ce " rien » érigé comme ultime refuge dans un désert $\mathrm{d}$ 'abandon et de désespoir, cette mort ci-gisant à même la vie, n'est-ce pas là la survivance du fantôme psychique de l'enfant mort ? Pour un peu, on prendrait le risque d'invoquer le mot « réincarnation » ou plus exactement le retour du cadavre en personne...

Les travaux de N. Abraham et M. Torok sur le «travail du fantôme ${ }^{13}$ » témoignent de ce pas, radicalement franchi jusqu'à en généraliser la portée à l'ensemble du champ psychopathologique, jusqu'à en tirer aussi toutes les conséquences, systématiques, pour la pratique analytique. À postuler à la source de toute pathologie l'action d'un fantôme enterré, c'est-à-dire incorporé tout rond, si l'on ose dire, et donc conservé intact et intégral comme embaumé, la visée de l'investigation et la pratique analytique se résument à une seule injonction : "Cherchez le fantôme. » Pour en être réduit à le reconnaître puis à le faire sortir de la psyché du patient, le geste analytique rejoint celui de l'exorciste!

Cette prise de position n'a pas manqué de susciter de nombreuses critiques ou réserves. Mais ce n'est pas au nom de l'impossibilité de trouver des archives du fantôme, ni de crainte que le sol de la réalité se dérobe sous les pieds de la psychanalyse. L'idée de la possession, directe et complète, de la psyché par le fantôme, accomplie sans autre forme de procès, présente l'attrait d'une ligne droite, claire et nette. Tant pis pour les brisures et les courbes enchevêtrées, elle fait l'économie des modes de transmission, singuliers et variables, et de strates fantasmatiques mobilisées, multiples et changeantes au gré des aléas de la vie psychique. Prenons le cas de l'enfant dit de remplacement. Ce n'est pas simplement d'être identifié à un mort que l'on peut souffrir. Comme chacun de nous vient toujours après, il est impossible qu'on

13. «Un dire enterré d'un parent devient chez l'enfant un mort sans sépulture. Ce fantôme inconnu revient alors depuis l'inconscient et exerce sa hantise, en induisant phobies, folies, obsessions. Son effet peut aller jusqu'à traverser des générations et déterminer le destin d'une lignée ", op. cit., p. 297. 
trouve une place vierge de toute ombre. Toute existence recèle en elle une "vie posthume », pour ainsi dire. Nul besoin de porter, en toutes lettres, le nom d'un mort. On n'en tombe pas forcément malade pour autant, bien au contraire, $c^{\prime}$ est même l'inverse qui s'avère éminemment pathogène : il suffit d'observer les effets aliénants du fantasme de parthénogenèse dont l'enfant est l'objet ou le support.

Le caractère éventuellement pathogène de la place de l'enfant de remplacement réside dans le fait qu'il est soumis à un mouvement antagoniste de la psyché parentale endeuillée : sa présence témoigne de l'intraitable perte dont elle est pourtant sommée d'annuler l'événement et de conjurer l'avènement ; il se doit alors d'être immortel ou presque, c'est-à-dire vivant perpétuellement et présent continuellement. Interdit de disparaître, de s'absenter, de se cacher, même en pensée et même par jeu. No play to hide and seek. " Tu ne dois pas disparaître comme ça, tu le sais très bien, tu le sais très bien ", cri de détresse d'une mère lancé à son enfant qui vient de jouer au disparu. Disparaître comme ça, pour rien. Un moment de trou noir pour la psyché maternelle occupée par son premier enfant, mort. Mais pour l'enfant, celui qui tente de survivre à l'enfant mort, ce jeu agi, comme le montre Nora Kurts ${ }^{14}$, est l'aboutissement et l'expression d'un travail d'investissement de l'absence, long et laborieux. Un plaisir enfin risqué, plaisir de jouer à partivoilà, si précieux pour la construction et la conservation de l'espace interne. Que de gens ont évité de se suicider en se contentant de déchirer leur photographie, comme le disait Jules Renard avec son humour noir ! Jouer à faire le mort comme pour jouer de la disparition, jouer à l'absent comme pour se jouer de l'absence. Le fameux jeu de la bobine, on le sait, tire son exemplaire vertu symbolisatrice, entre autres, de l'identification à la mère absente que représentent indistinctement la bobine qui disparaît et l'enfant qui la jette au loin. Or c'est, paradoxalement, cette dynamique de l'identification, active, à l'absent dont se trouve privé l'enfant de remplacement! Se défaire de l'ombre de l'enfant mort suppose de se l'approprier ; aussi minime soit-il, existe donc un écart entre " être assigné à » et " s'identifier à », entre la forme passive et le mouvement réfléchi, qu'il importe d'apprécier. Car, sans être assurément établi, l'écart peut être significatif d'une tentative de mise au-dedans que veut dire le fantasme. Là on peut découvrir, soutenues par la mobilisation transférentielle, toute la polysémie et l'épaisseur fantasmatiques de la figure de l'enfant mort. Par exemple, celle-ci peut se faire obstinément insistante dans un paysage psychique tout bruissant de la rumeur œdipienne. L'enfant mort y noue alors jusqu'à l'enchevêtrement plusieurs fils fantasmatiques et identificatoires : crime et châtiment par la même voie, meurtrier coupable et

14. N. Kurts, "L'enfant qui ne savait pas jouer ", Psychiatrie de l'enfant, XXXVI, 2, 1993, p. $551-554$. 
victime innocente en une seule figure, réparation et répétition par le même geste fantasmatique.

Qu'en est-il alors de la figure de l'enfant mort qui, comme on l'a rappelé plus haut, hante électivement une contrée psychique immobile et nappée de brumes épaisses, aux confins de la mélancolie ? Dans ce tréfonds de la psyché, là où les mots ont la plus grande peine à demeurer vivants, où la quête radicale de clôture narcissique menace d'abolir la frontière entre vivant et mort, l'enfant mort est une figure à la fois figée et évanescente. Un arrêt sur image, en miroir avec l'immobilité mélancolique, mais une image tout de même. Envisagée dans sa valeur fantasmatique, la figure de l'enfant mort réalise le paradoxe d'une tentative de mise en forme de ce qui tend à son abolition : quête de fusion jusqu'à l'uniforme, quête de confusion jusqu'à l'informe ${ }^{15}$. Une représentation limite à la mesure du paradoxe de la visée mélancolique : tendre à la passivité totale, pour en annuler et maîtriser $l^{\prime}$ excès pulsionnel annihilant ${ }^{16}$.

On pourrait soutenir que l'enfant mort figure la passivité dont la violence effractante déborde le traitement par la fantasmatique masochiste du type " on bat un enfant » et sollicite, sans l'accomplir complètement, la tentation mélancolique de l'extinction des sources pulsionnelles. La position tendue et inconfortable de la figure de l'enfant mort entre masochisme et mélancolie constitue une difficulté certaine, mais pas seulement. Elle peut aussi indiquer une ouverture de potentialité fantasmatique plus grande. En tout cas, elle permet d'observer et d'interroger comment certains destins de la féminité se cherchent à l'ombre de la figure de l'enfant mort, au-delà de la référence à une réalité historique vécue ou transmise.

\section{L'ENFANT MORT ET LA FÉMINITÉ}

«Tout ce que je vois, c'est une femme morte, une femme à la morgue, on ne voit pas son visage. » Ces mots sont ceux arrachés à un corps de femme en train de sombrer dans les flots de larmes. Les mots rescapés. Senta vient de risquer, pour la première fois, quelques mots sur le viol subi à 15 ans : sortie de l'école, une connaissance de la mère, chemin du retour inhabituel, trois hommes et une voiture, quelque part près du bois de son village ; elle ne s'est pas débattue, même pas de cris d'appel à l'aide, n'était cette supplication dans sa tête, déjà détachée de son corps : «Ce n'est pas possible, mon

15. Pour une vue d'ensemble de configurations pathologiques mettant en jeu la problématique de l'informe, voir Sylvie Le Poulichet, Psychanalyse de l'informe. Dépersonnalisations, addictions, traumatismes, Paris, Flammarion, 2003.

16. C. Chabert, "Masochisme ou mélancolie ? ", dans Féminin mélancolique, Paris, PUF, coll. " Petite bibliothèque de psychanalyse », 2003. Voir aussi G. Rosolato, "L'axe narcissique des dépressions ", dans La relation d'inconnu, Paris, Gallimard, 1978. 
Dieu, venez, que ça s'arrête. »C'était il y a longtemps, dans son pays natal, avant l'éclatement de la guerre civile ravageuse. Pour Senta, la guerre était là depuis toujours, quotidienne : violence, maltraitance, alcool, sexe, dénuement, abandon, errance. Mais ce jour-là, en séance, ce n'est pas seulement l'évocation du viol qui la plonge dans cette profonde détresse, mais quelque chose qui vient d'avoir lieu devant et en elle, ici et maintenant : voir le regard maternel qui ne voit rien. Ivre morte comme à ses habitudes, la mère ne s'est rendu compte de rien, ni de l'absence de sa fille, ni de l'état dans lequel Senta était rentrée à la maison : " Elle n'a rien vu, rien. » Puis elle pleure, elle fond, se dissout dans ses larmes, devant moi, silencieuse et impuissante, qu'elle ne voit pas. Mon silence revient à ne rien voir, à renvoyer au néant ce qui m'est donné à voir. Un silence de mort en écho. Mais comment m'en dégager pour lui restituer quelque chose de ce qui se passe ? Peut-on demander à quelqu'un qui crie au secours : «Qu'entendez-vous par là ? " ? C'est tout de même quelque chose d'aussi absurde que j'ai tenté, si ce n'est que je lui ai parlé du regard maternel indifférent et de mon silence impuissant, avant de lui demander ce qu'elle voyait en pleurant.

"Une femme morte, à la morgue, sans visage. » Cette image brutale et saisissante, ouverte par l'actualisation transférentielle de la détresse et de la passivité, appelle à la mémoire d'autres scènes, notamment une scène, un autre souvenir d'effraction : la petite fille dort dans un lit qui n'est pas le sien, probablement celui d'un amant de passage de la mère. Une nuit ordinaire des pérégrinations sexuelles de la mère à qui la compagnie de sa fille de 7 ans sert d'alibi et de gage d'innocence. Seulement, au milieu de cette nuit-là, Senta se retrouve dans le lit, à côté du couple de corps ivres, emmêlés dans un mouvement de lutte sexuelle, qui la tire du sommeil. L'enfant dort, et comme si elle n'était pas là pour ces adultes maltraitants par leur excitation et cruels par leur indifférence. Pendant une longue période qui a suivi cet épisode traumatique, Senta, honteuse et coupable, devient mutique, comme frappée de silence de mort.

"Une femme morte, à la morgue, sans visage » est une version de « on tue un enfant ». Cette image violente condense effraction sexuelle, passivité de la position féminine et détresse infantile. Elle témoigne aussi d'une résistance opposée par un narcissisme mortellement blessé et de la menace de la mélancolie triomphale : on ne peut plus violer ni abandonner une morte, plus rien ne peut lui arriver, c'est fait, advenu déjà et pour toujours, morte, sans visage, invulnérable, abritée dans une chambre froide comme un corps inanimé, à l'image de ce regard déserté par la lumière, un regard mort qui ne voit rien. Femme morte ou enfant mort ? Peu importe la différence de générations dans cette région psychique où les vivants et les morts se confondent. Pour Senta, l'analyse est comme la sortie d'un état de coma. De ce réveil long, 
incertain et douloureux, l'apparition de la figure de l'enfant mort, le déploiement de toute son épaisseur fantasmatique marquent paradoxalement un des premiers pas. L'image de la «femme morte » est prolongée et suivie d'un état dépressif ponctué par les plaintes somatiques et le cri, enfin adressé : «J'ai mal. » Comme si la réanimation psychique et la réappropriation du corps se retrouvaient condensées dans l'expérience de la douleur, comme si, quand ce qui est mort revient à la vie, cela ne pouvait pas manquer de faire mal...

La figure de l'enfant mort semble ainsi particulièrement sollicitée dans l'élaboration de la féminité dont la puissance traumatique tient en échec le fantasme masochiste - celui qui conjugue jouissance et effraction - et sur laquelle pèse donc la menace de la solution mélancolique. Elle témoigne d'un destin de la féminité suspendu entre masochisme et mélancolie. Lors de son émergence perceptible ou rendue possible, on gagnerait à la maintenir sur cette ligne de crête, à prendre le temps de ne pas la réduire à la manifestation d'un fantasme narcissique, ni au triomphe de l'immobilité mélancolique, encore moins à l'existence d'un fantôme qu'il suffirait, pour le déloger, d'exhumer. Il arrive que le feu couve sous ses cendres et le fantôme révèle une vie fantasmatique insoupçonnée. Mort à jamais ? Qui peut le dire?

\section{BIBLIOGRAPHIE}

ABraham, N. ; TOROK, M. 1978. L'écorce et le noyau, Paris, Flammarion, 1987.

ANDRÉ, J. 2008. Folies minuscules, Paris, Gallimard.

CHABERT, C. 2003. Le féminin mélancolique, Paris, PUF, coll. " Petite bibliothèque de psychanalyse ».

DARRIEUSSECQ, M. 2007. Tom est mort, Paris, POL.

FREUD, S. 1907. Le délire et les rêves dans la Gradiva de W. Jensen, Paris, Gallimard, 1986.

FREUD, S. 1909. "Analyse de la phobie d'un garçon de cinq ans », dans CEuvres complètes IX, Paris, PUF, 1998.

LAurens, C. 1995. Philippe, Paris, POL.

Le Poulichet, S. 2003. Psychanalyse de l'informe. Dépersonnalisations, addictions, traumatismes, Paris, Flammarion.

PONTALIS, J.-B. 1997. Ce temps qui ne passe pas, Paris, Gallimard.

Quignard, P. 1994. Le sexe et l'effroi, Paris, Gallimard, coll. « Folio ».

QUignaRD, P. 2007. La nuit sexuelle, Paris, Flammarion.

Rosolato, G. 1978. "L'axe narcissique des dépressions », dans La relation d'inconnu, Paris, Gallimard.

Résumé

Le fantôme serait-il devenu l'ombre de lui-même, irrémédiablement ? Ombre enkystée aux accents mélancoliques, spectre errant sous les auspices du destin, telle est la vision du fantôme particulièrement sensible et imposante dans la clinique des 
dépressions et dans la problématique du transgénérationnel. À travers la figure de l'enfant mort régulièrement repérable au croisement de ces deux problématiques, l'auteur propose quelques considérations qui mettent en évidence les rapports multiples et complexes entre fantôme et fantasme.

Mots-clés

Enfant mort, fantôme, fantasme, topique, narcissisme, mélancolie, transgénérationnel, sexualité féminine.

THE DEAD CHILD FIGURE BETWEEN PHANTOM AND FANTASY

\section{Summary}

All of that remains of the phantom might be just the shadow of itself ? Currently considered as a melancholic shadow enclosed, or as the haunting fate, the phantom concerns in particular two psychopathology fields : depression and intergenerational transmission. Through a study of an interesting figure crossing both of them - the dead child -, the author submits several ideas about the complex relationship between phantom and fantasy.

Keywords

Dead child, phantom, fantasy, topography, narcissism, melancholy, intergenerational transmission, female sexuality. 